\title{
An Overview of Meson-Nuclear Physics
}

\author{
Anthony W. Thomas \\ CSSM, School of Chemistry and Physics, University of Adelaide \\ Adelaide SA 5005, Australia
}

\begin{abstract}
In this opening talk at MENU2010 we outline some of the key achievements in the field over the past few years as well as some of its major challenges and opportunities.

Keywords: QCD, strange quarks, meson and baryon spectroscopy, hadron structure in medium, Standard Model tests, dark matter, new facilities

PACS: 12.38.-t, 11.30.-j, 12.39.-x, 13.25.-k, 13.30.Eg, 13.40.-f, 13.75.-n, 14.20.-c, 21.30.-x, 21.65.-f, 24.80.+y, 24.85.+p, 25.30.-c, 26.60.-c
\end{abstract}

\section{INTRODUCTION}

This conference covers an extremely broad range of topics and in just a few pages it is impossible to even touch on all the areas which will be discussed. We have chosen to summarise just one area where there has recently been impressive progress, namely our quantitative understanding of strangeness in the nucleon. We also discuss a couple of examples where theoretical progress is of direct importance for future experiments as well as for the interpretation of hitherto anomalous results. Finally we make some remarks on meson and baryon spectroscopy and the exciting array of new facilities that are coming on-line in the near future.

\section{STRANGENESS}

The absence of strange valence quarks in the nucleon means that they can only contribute to hadron properties through the creation of virtual quark-anti-quark pairs and thus the accurate test of their role plays a role for QCD analogous to that of the Lamb shift in QED. Some 5 years ago the use of indirect techniques [1], involving lattice QCD, chiral extrapolation and measured baryon properties led to extremely accurate determinations of the strange magnetic moment and charge radius of the proton [2,3]. Just last year the magnetic moment calculation was beautifully confirmed by the first direct lattice calculation [4]. Contrary to much speculation over the preceding decade, these contributions turn out to be quantitatively quite small - below $1 \%$ for both the magnetic moment and the mean-square radius. The experimental results for the strange form factors of the proton $[5,6,7,8]$ are in remarkable agreement with the theoretical calculations [9], although for now the errors are perhaps an order of magnitude larger.

Studies of hadron structure as a function of quark mass have provided a very natural explanation of these results [10], namely that the finite size of the source of the meson clouds associated with chiral symmetry naturally suppress all Goldstone boson loops for meson masses above around $0.4 \mathrm{GeV}$ [11]. The physical kaon mass falls above this limit and is naturally suppressed. The exploitation of this remarkable feature of nucleon structure through finite range regulated effective field theory has also led to a much more precise and again astonishingly small result for another property of strange quarks in the proton, namely the strange sigma commutator. Young and Thomas recently reported a value around $30 \mathrm{MeV}$ [12], which is an order of magnitude smaller than commonly used and believed. This result is also in excellent agreement with direct calculations that appeared around the same time [13, 14].

This new value for $\sigma_{s}$ not only has consequences for speculation about possible kaon condensation but surprisingly it is also extremely important for searches for neutralinos - the favourite "beyond-the-Standard-Model" candidate for dark matter. Within the minimal supersymmetric extension it turns out that the biggest contribution to the neutralinonucleon spin independent cross section came through $\sigma_{s}$, until this new result. As discussed by Giedt et al. [15], the new value solves a long-standing problem of increasing the accuracy of predictions of these cross sections but it also leads to values at least an order of magnitude smaller than hitherto expected. This has major implications for dark matter searches as well as for the interpetation of existing observations. 


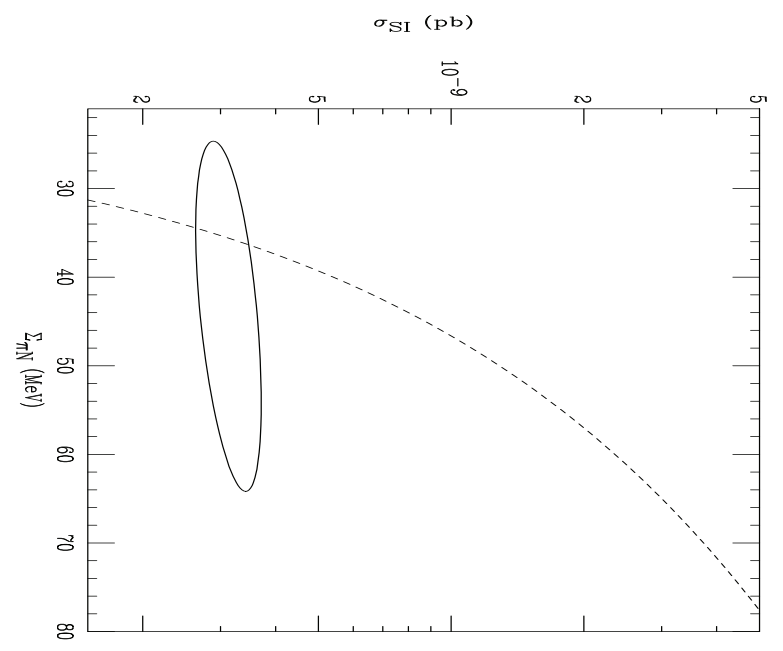

FIGURE 1. Illustration of the improvement in the precision with which the neutralino-nucleon cross section can be calculated within a particular minimal supersymmetric extension of the Standard Model when the strange quark sigma commutator is taken from the recent analysis of lattice data by Giedt et al. [15], rather than through the $\pi N$ sigma commutator. Note that although the precision is much improved the absolute cross section is very much at the lower end of the range.

A final issue on which there has also been substantial recent progress concerns the parton distribution functions (pdfs) for strange quarks [17]. This is extremely important in connection with the NuTeV anomaly [16], where an asymmetry between $s$ and $\bar{s}[18]$ quarks can provide a potentially large correction. Global analyses of pdfs seem to indicate a positive value for $\left\langle x s^{-}\right\rangle$, but the level of precision needs substantial improvement. For this particular issue, lattice QCD seems unlikely to make a significant contribution in the near future. However, for the combination $\langle x(s+\bar{s})\rangle$, the Kentucky group has recently been able to obtain quite an accurate value, namely $2.7 \pm 0.3 \%$ [19]. This may increase somewhat when the light quark masses take more physical values but the calculation nevertheless represents substantial progress.

\section{SPIN DEPENDENDENT PHENOMENA}

There continues to be a great deal of effort devoted to clarifying the relative importance of quark and gluon spin and orbital angular momentum in defining the spin of the proton. Data from RHIC has established that the total spin carried by gluons is less than about one half at a scale of a few $\mathrm{GeV}^{2}[20,21]$, and no doubt this will be pinned down even more precisely over the next few years. We already know that it is nowhere near large enough to play a significant role in resolving the famous spin crisis, where almost certainly it is the relativistic quark motion combined with gluon exchange and chiral symmetry that leads to the replacement of spin by quark orbital angular momentum [22, 23]. The debate over how best to define quark and gluon orbital angular momentum continues with vigour $[24,25]$ and this will be a topic for discussion at this meeting.

Within the experimental community there is tremendous excitement over the host of new observables which promise to bring new light to the study of hadron structure [26]. This includes a number of measurements involving semiinclusive hadron production which will enable the determination of GPDs and TMDs [27]. On the theoretical side, we note the tremendous progress made in lattice QCD to measure moments of the GPDs relevant to the proton spin problem [28]. Critical issues for the near future include the need to develop a deep understanding of the systematic errors associated with finite volume corrections and extrapolations in both pion mass and momentum transfer needed to accurately pin down the quark orbital angular momentum $[29,30]$.

\section{Hadrons in-medium}

The nuclear community shows conflicting attitudes to the role of QCD in providing the fundamental explanation of the properties of atomic nuclei. For some QCD exists simply to give us chiral symmetry and nuclear structure can 


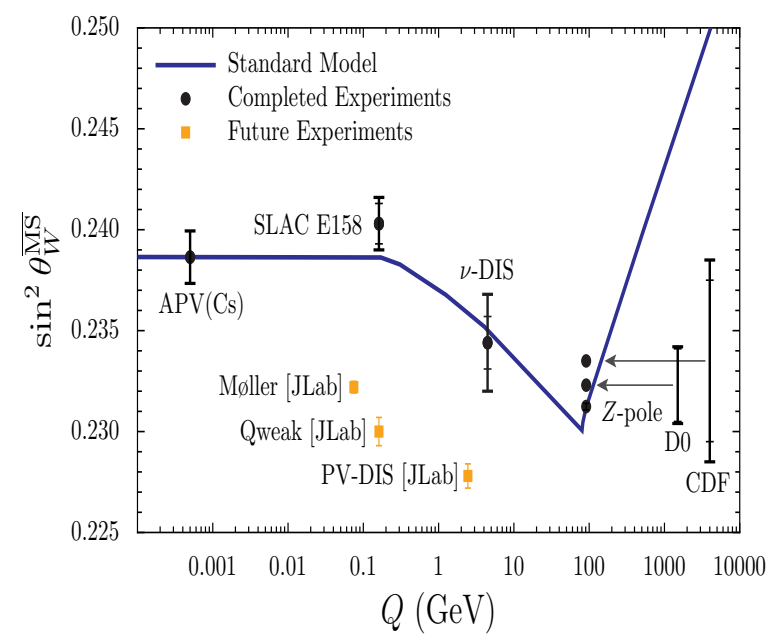

FIGURE 2. The curve represents the running of $\sin ^{2} \theta_{W}$ in the $\bar{M} S$ renormalization scheme. The $Z$-pole point represents the combined results of six LEP and SLC experiments, while the CDF and D0 collaboration results (at the Z-pole) and the SLAC E158 result, are labelled accordingly. The atomic parity violating (APV) result has been shifted from $Q^{2}=0$ for clarity. The inner error bars represent the statistical uncertainty and the outer error bars the total uncertainty - see Ref. [42] for details and associated references.

then be computed in terms of a number of low energy constants fixed by data. This is a more formal and systematic version of the older approach, still very much in favour, where one calculates nuclear properties using two-, three- and more-body forces, the former fitted to $\mathrm{NN}$ data while the latter are fit to chosen sets of nuclear data. The most modern expression of this approach involves the use of the techniques of lattice QCD to compute the interaction of two and three hadrons directly from QCD [31,32]. This is an ambitious long-term program, with current calculations limited to rather small lattice volumes. Nevertheless, as we shall see at the conference some remarkable results have already been obtained.

An alternate approach begins with the realization that at some density (perhaps 3 to 10 times nuclear matter density) nuclear matter will make a transition to quark matter - a phase transition which may have dramatic effects on the observable properties of neutron stars. This suggests that one might begin to build a theory of the nuclear manybody system starting with a description of hadron structure at the quark level and then considering the self-consistent modification of that structure in a nuclear medium. This is the approach taken within the QMC (quark-meson coupling) model $[33,34]$. A remarkable advantage of this approach is that no new parameters are needed to calculate the effective density dependent forces [35] between any hadrons whose quark structure is known. Indeed, it has been possible to develop a remarkably successful derivation of realistic Skyrme forces $[35,36]$ for comparison with low energy nuclear phenomenology - while the fully relativistic underlying theory successfully predicts key features of hypernuclear physics and allows the study of the appearance of hyperons in dense matter [37]. Most recently, this model has also been used to study the possibility of binding of charm-anti-charm mesons in matter [38], with results very similar to those coming from direct lattice QCD simulations [39].

The QMC model has the additional advantage that one can address not only those low energy properties such as binding energies and charge densities but it can also be used to calculate the nuclear modification of the deep inelastic structure functions. Extensions of the QMC approach based upon a covariant, confining version of the NJL model have produced a satisfactory description of the EMC effect in finite nuclei [40]. It has also produced the remarkable prediction that should motivate quite a bit of experimental effort, namely that the EMC effect for the spin structure function should be roughly twice as large as the unpolarised EMC effect [40]. Another recent realisation of critical importance if one intends to use deep inelastic scattering data on nuclei in any way that assumes some sort of flavour symmetry, is that there will be a component of the EMC effect which is isovector in nature if one has a target with $N \neq Z$ [41]. Most importantly, because the EMC effect involves a change in the structure of the bound nucleon, that isovector EMC correction will persist even if one derives data for an effectively isoscalar nucleus by subtracting the contribution of the excess neutrons. A very important example of this is the corresponding correction to the PaschosWolfenstein relation in the $\mathrm{NuTeV}$ determination of $\sin ^{2} \theta_{W}$. Figure 2 shows the result of a recent reanalysis of the $\mathrm{NuTeV}$ anomaly [42] in which this correction was applied to the data along with a correction for genuine charge 
symmetry violation associated with the mass difference between $u$ and $d$ quarks.

\section{HADRON SPECTROSCOPY}

Many presentations at this meeting will address recent developments in hadron spectroscopy. The Excited Baryon Analysis Center at JLab is now producing detailed coupled channels calculations for two pion production as well as kaon production in pion and photon induced reactions [43, 44]. In close collaboration with other groups at Jülich, Bonn and Mainz this has already clarified our understanding of some well established resonances [45]. It has also established a sound basis for the extraction of information on any new states that may appear in the mass region above 1.8-2.0 GeV, where old quark model ideas suggest that there should be more states than have hitherto been seen. This coupled channel analysis is being challenged by a considerable quantity of new, high quality data that will help to answer these questions.

On the theoretical side, lattice QCD has again proven to be a critical player. In particular, the JLab group has remarkable new results for both charm quark [46] and light quark [47] systems, with a careful blend of group theory and lattice techniques now allowing the clear identification of a large number of excited states for each choice of spin and parity. As the masses of the light quarks in these simulations approach the physical values more closely it will be necessary to deal with effects of channel coupling here too.

\section{NEW FACILITIES}

Across the world this field either has or soon will have the benefit of a large number of major new experimental facilities and there will be considerable discussion of their capabilities at this conference. At this time the most noteworthy is, of course, JPARC in Japan, which only recently delivered its first beam. While the beam intensity is currently low and the energy currently $30 \mathrm{GeV}$ rather than the ultimate $50 \mathrm{GeV}$ the accelerator is already delivering a neutrino beam to the Kamiokande detector and the high momentum kaon line is ready for experiments. The first of those experiments, using pions rather than kaons, will take one more look at the infamous pentaquark. This object, which would add remarkably to our zoo of fundamental particles if confirmed, currently looks rather sick, with only one of the initial groups that reported a sighting still claiming to have evidence for its existence. Hopefully we will have news of that test at the next meeting.

Apart from its role in neutrino oscillations, the facilities at JPARC are ideally suited for extending our knowledge of hypernuclei dramatically. Of particular importance is the discovery of $\Xi$-hypernuclei, which are expected, within the QMC model discussed earlier, to be bound by bound by up to $20 \mathrm{MeV}$ [48]. Establishing their existence and eventually the systematics of their binding and structure is not only important because it will establish a totally new area of study in nuclear structure but because of its importance for the properties of dense matter, as found for example in neutron stars. Certainly, if $\Xi$-hypernuclei are confirmed it will be difficult to avoid the conclusion that the $\Xi^{-}$should appear at densities not far above $2 \rho_{0}$ in matter that is in $\beta$-equilibrium. Their appearance, in turn, has the effect of dramatically softening the equation of state and hence the observable properties of neutron stars. Finally, we mention the promise of Drell-Yan for studies of the flavour structure of hadrons and nuclei as well as offering new types of spin-dependent measurements in which one can more easily detect processes which violate chiral symmetry.

Two future facilities, due to operate at a similar date, which will also undoubtedly be the source of intense interest are the $12 \mathrm{GeV}$ upgrade at JLab (just a few kilometers away from this meeting) and the new facilities at GSI-FAIR. Hadron spectroscopy, especially the study of the existence and properties of the exotic mesons predicted within QCD, should be transformed by the Gluex experiment in Hall D. (Though we should also mention the important studies currently underway at COMPASS at CERN.) Critical to the success of this experiment, which should be able to access states up to as much as $2.6-2.8 \mathrm{GeV}$, is the dedicated $9 \mathrm{GeV}$ photon beam with high linear polarisation for which Hall D was built, as well as the extremely high acceptance of the purpose built detector. GSI-FAIR also aims to produce and study $\Xi$-hypernuclei and both new facilities, as well as JPARC, should be able to develop the spectroscopy of strange baryons to a new level.

The development of a circulating anti-proton beam at GSI-FAIR will probably come a little after the opening of this new facility but the set of experiments already proposed for it, including precision charmonium spectroscopy, is very exciting. We leave the many other possibilities opened by these facilities for discussion at the meeting, except to emphasise the kinematic range opened for the study of the spin and flavour dependence of the parton distribution functions, as well as semi-inclusive deep inelastic processes by the $12 \mathrm{GeV}$ upgrade. 
We also expect that there will be lively discussion over the long term plans to build one or more electron-ion colliders in Europe and the United States. The very ambitious plans at BNL and JLab will come to a focus in the next long range plan for the United States, while the somewhat more modest plans at GSI-FAIR are still under development. Certainly the plans for an electron-ion collider at BNL or JLab offer a tremendous opportunity to look for non-perturbative gluonic effects as well as allowing one to probe the spin and flavour structure of matter at a deeper level than possible before. One should also mention the possibility of installing an electron accelerator at CERN so that one could have electron-ion collisions at the $\mathrm{LHC}$, the $\mathrm{LHeC}$ project. This is extremely ambitious in terms of cost and energy reach (although it has no polarisation) but clearly would be motivated by discoveries at the LHC itself and therefore a decision must await the first discoveries there.

\section{SUMMARY}

In this brief welcome I have been able to do little more than touch on a few of the exciting physics challenges that will be discussed in detail at this meeting, as well as very briefly highlighting the wonderful array of new facilities that will be crucial to the resolution of those challenges. I wish all participants the best for a productive meeting!

\section{ACKNOWLEDGMENTS}

This work was supported by the Australian Research Council through an Australian Laureate Fellowship and by the University of Adelaide.

\section{REFERENCES}

1. D. B. Leinweber, A. W. Thomas, Phys. Rev. D62, 074505 (2000). [hep-lat/9912052].

2. D. B. Leinweber, S. Boinepalli, I. C. Cloet et al., Phys. Rev. Lett. 94, 212001 (2005). [hep-lat/0406002].

3. D. B. Leinweber, S. Boinepalli, A. W. Thomas et al., Phys. Rev. Lett. 97, 022001 (2006). [hep-lat/0601025].

4. T. Doi, M. Deka, S. -J. Dong et al., [arXiv:0910.2687 [hep-lat]].

5. D. Androic et al. [ G0 Collaboration ], Phys. Rev. Lett. 104, 012001 (2010). [arXiv:0909.5107 [nucl-ex]].

6. A. Acha et al. [ HAPPEX Collaboration ], Phys. Rev. Lett. 98, 032301 (2007). [nucl-ex/0609002].

7. K. A. Aniol et al. [ HAPPEX Collaboration ], Phys. Rev. Lett. 96, 022003 (2006). [nucl-ex/0506010].

8. F. E. Maas et al. [ A4 Collaboration ], Phys. Rev. Lett. 93, 022002 (2004). [nucl-ex/0401019].

9. R. D. Young, J. Roche, R. D. Carlini et al., Phys. Rev. Lett. 97, 102002 (2006). [nucl-ex/0604010].

10. A. W. Thomas, R. D. Young, D. B. Leinweber, [nucl-th/0509082].

11. R. D. Young, D. B. Leinweber, A. W. Thomas, Prog. Part. Nucl. Phys. 50, 399-417 (2003). [hep-lat/0212031

12. R. D. Young, A. W. Thomas, Phys. Rev. D81, 014503 (2010). [arXiv:0901.3310 [hep-lat]].

13. D. Toussaint et al. [ MILC Collaboration ], Phys. Rev. Lett. 103, 122002 (2009). [arXiv:0905.2432 [hep-lat]].

14. H. Ohki, H. Fukaya, S. Hashimoto et al., Phys. Rev. D78, 054502 (2008). [arXiv:0806.4744 [hep-lat]].

15. J. Giedt, A. W. Thomas, R. D. Young, Phys. Rev. Lett. 103, 201802 (2009). [arXiv:0907.4177 [hep-ph]].

16. G. P. Zeller et al. [ NuTeV Collaboration ], Phys. Rev. Lett. 88, 091802 (2002). [hep-ex/0110059].

17. S. Alekhin, S. A. Kulagin, R. Petti, Phys. Lett. B675, 433-440 (2009). [arXiv:0812.4448 [hep-ph]].

18. A. I. Signal, A. W. Thomas, Phys. Lett. B191, 205 (1987).

19. M. Deka, T. Streuer, T. Doi et al., Phys. Rev. D79, 094502 (2009). [arXiv:0811.1779 [hep-ph]].

20. A. Adare et al. [ PHENIX Collaboration ], Phys. Rev. Lett. 103, 012003 (2009). [arXiv:0810.0694 [hep-ex]].

21. B. I. Abelev et al. [ STAR Collaboration ], Phys. Rev. Lett. 100, 232003 (2008). [arXiv:0710.2048 [hep-ex]].

22. F. Myhrer, A. W. Thomas, Phys. Lett. B663, 302-305 (2008). [arXiv:0709.4067 [hep-ph]].

23. A. W. Thomas, Phys. Rev. Lett. 101, 102003 (2008). [arXiv:0803.2775 [hep-ph]].

24. M. Wakamatsu, Phys. Rev. D81, 114010 (2010). [arXiv:1004.0268 [hep-ph]].

25. X. -S. Chen, W. -M. Sun, X. -F. Lu et al., Phys. Rev. Lett. 103, 062001 (2009). [arXiv:0904.0321 [hep-ph]].

26. A. V. Belitsky, A. V. Radyushkin, Phys. Rept. 418, 1-387 (2005). [hep-ph/0504030].

27. A. Afanasev, M. Anselmino, H. Avakian et al., [hep-ph/0703288 [HEP-PH]].

28. J. D. Bratt et al. [ LHPC Collaboration ], [arXiv:1001.3620 [hep-lat]].

29. A. W. Thomas, ACasey, H. H. Matevosyan, Int. J. Mod. Phys. A25, 4149-4162 (2010).

30. P. Wang, A. W. Thomas, Phys. Rev. D81, 114015 (2010). [arXiv:1003.0957 [hep-ph]].

31. S. R. Beane, W. Detmold, K. Orginos et al., [arXiv:1004.2935 [hep-lat]].

32. T. Hatsuda [ HAL QCD Collaboration ], PoS CD09, 068 (2009). [arXiv:0909.5637 [hep-lat]].

33. P. A. M. Guichon, K. Saito, E. N. Rodionov et al., Nucl. Phys. A601, 349-379 (1996). [nucl-th/9509034]. 
34. K. Saito, K. Tsushima, A. W. Thomas, Prog. Part. Nucl. Phys. 58 (2007) 1-167. [hep-ph/0506314].

35. P. A. M. Guichon, H. H. Matevosyan, N. Sandulescu et al., Nucl. Phys. A772, 1-19 (2006). [nucl-th/0603044].

36. P. A. M. Guichon, A. W. Thomas, Phys. Rev. Lett. 93, 132502 (2004). [nucl-th/0402064].

37. P. A. M. Guichon, A. W. Thomas, K. Tsushima, Nucl. Phys. A814, 66-73 (2008). [arXiv:0712.1925 [nucl-th]].

38. G. Krein, A. W. Thomas, K. Tsushima, “J/ $\Psi$ mass shift in nuclear matter," [arXiv:1007.2220 [nucl-th]].

39. K. Yokokawa, S. Sasaki, T. Hatsuda et al., Phys. Rev. D74, 034504 (2006). [hep-lat/0605009].

40. I. C. Cloet, W. Bentz, A. W. Thomas, Phys. Lett. B642, 210-217 (2006). [nucl-th/0605061].

41. I. C. Cloet, W. Bentz, A. W. Thomas, Phys. Rev. Lett. 102, 252301 (2009). [arXiv:0901.3559 [nucl-th]].

42. W. Bentz, I. C. Cloet, J. T. Londergan et al., Phys. Lett. B693, 462-466 (2010). [arXiv:0908.3198 [nucl-th]].

43. A. M. Sandorfi, S. Hoblit, H. Kamano et al., [arXiv:0912.3505 [nucl-th]].

44. T. S. H. Lee, Int. J. Mod. Phys. E18, 1215-1225 (2009).

45. S. X. Nakamura, H. Kamano, T. -S. H. Lee et al., [arXiv:1008.0214 [nucl-th]].

46. J. J. Dudek, R. Edwards, C. E. Thomas, Phys. Rev. D79, 094504 (2009). [arXiv:0902.2241 [hep-ph]].

47. J. J. Dudek, R. G. Edwards, M. J. Peardon et al., Phys. Rev. Lett. 103, 262001 (2009). [arXiv:0909.0200 [hep-ph]].

48. K. Tsushima, P. A. M. Guichon, R. Shyam et al., [arXiv:0903.5478 [nucl-th]]. 